\title{
An Analytical Approach for Design of Microstrip Patch (MsP)
}

\author{
R. J. Kavitha ${ }^{1}$, H. S. Aravind ${ }^{2}$ \\ ${ }^{1}$ Visvesvaraya Technological University, India \\ ${ }^{2}$ Department of Electronics \& Communication Engineer, JSSATE, India
}

\begin{tabular}{l}
\hline \hline Article Info \\
\hline Article history: \\
Received Mar 14, 2018 \\
Revised Jul 17, 2018 \\
Accepted Aug 5, 2018 \\
\hline
\end{tabular}

Keyword:

Computational cost

Microstrip patch antenna

Radiation coefficients

Reflection coefficients

Side lobes

\begin{abstract}
A reliable configuration of electromagnetic interactions for antenna design can yield an effective Microstrip patch (MsP) antenna. During its design, the antenna arrays involve issues with parameters (i.e., space, dimension, shape) adjustment. This problem can be tackled with an analytical approach which can help to bring better idea to design the antenna aaray. However, the realistic designs of antenna array are quite expensive while extracting computational accuracy. Thus, to have low cost computational accuracy various meta-heuristic (generic algorithm, partical swarm optimizarion) approaches are used and are considered as effective one in handling the pattern synthesis problems. Howeever, the use of meta-heuristic approaches demands thousands of functions to analyze the antenna design. This manuscript introduces an analytical approach for MsP antenna desing using MATLAB that brings optimization in handling the side lobes and optimizing the reflection as well as radiation responses. The outcomes of the design were analyzed with respect to reflection, radiation coefficients, side lobes and found effective at $10 \mathrm{GHz}$ as per computational cost is concern.
\end{abstract}

Copyright $@ 2018$ Institute of Advanced Engineering and Science. All rights reserved.

\section{Corresponding Author:}

R. J. Kavitha,

Visvesvaraya Technological University,

Belagavi, India.

kavitharjkkiran@gmail.com

\section{INTRODUCTION}

The Microstrip Patch (MsP) antenna arrays design demands a reliable electro-magnetic interactions (EmI) within antenna array structures to provision the requirements of the antenna design induced by array radiations and reflection responses [1]. The electro-magnetic interactions are consists of element environment, element coupling, substrate finite size, feeding impact etc. Such impacts can only be reliably accounted for design process through typically discrete, full-wave, electro-magnetic simulations mainly by using complete antenna array module [2].

The antenna array design involved with issues elements dimensions adjustment, array shape adjustment, array spacing adjustment, feeding location adjustment etc. In that regard, a mathematical approach can be considered as effective for significant way of antenna array design [3]. The real time implememtation of mathematical approach can leads to higher cost in antenna array design as it takes of more number of simulations iterations in array model [4]. The meta-heuristics mechanisms such as particle swarm optimization [5]; genetic algorithms [6] are outcomes with significant results which can handle the pattern synthesis issues [7]. The limitation of meta-heuristic mechanisms is that it needs thousands of functions for antenna design analysis. Hence, this paper aims to perform the accurate design of MsPantrnna by using an analytical approach for pattern synthesis. Finally, the design analysis is performed by considering the parameters like radiation, reflection coefficients, operating frequency and minimization of side lobes. The paper is organized with sections like reveiew of existing works (in section 2), design and implementation of proposed system (in section 3), results and analysis (in section 4) and conclusion (in section 5). 


\section{RELATED WORK}

The recent researches towards antenna design are reviewed in this section. A wast range of researches are exist towards the design of wideband antenna and are capable of handling the transmission issues and offer high speed communication. A survey work towards the design of MsP antenna and its contribution towards communication system is found in Kavitaand Aravinda (2017) [8] that offers existing research gap in MsP antenna technology. Similarly, Tang et al. (2016) [9] presented a strip-helical antenna system with a parasitic circular patch for circular polarization which are capable of working like an impedance bandwidth.

Further, Salih et al. [10] gave a dual-band patch antenna for small form factor devices. This antenna was fabricated over Rogers (RO4350) board, the measured result have a good agreement with simulated ones. A dual-band circularly polarized antennas have been receiving much attention due to their distinct feature of single layer and single feeds. There are two different dissimilar radiators and two pairs of degenerate's mode TM01/TM10 and TM03/TM30 modes. In order to minimize the cost, miniaturised size, and easy integration author Zahang et al. (2016) [11] introduced a dual-frequency band polarisation method which achieves the radition about 7.0and 5.9 in the two bands. In Katyal et al. (2016) [12], a transmissionline equivalent circut technique is presented for analysis of multilayered MsP antenna.The performance of the technique is validated by analysis of broadband antenna and found that the proposed method is capable for quick circuir level imitation and optimization. In the study of different type of printed antennas having different type of patch like, rectangular, square, triangular, circular, elliptical are suitable for $60 \mathrm{HZ}$ wairless application.

For reduce over all size and to avoid lossymillimetre-wave connectors author Hannachi et al. [13] has proposed a keysight technologies, this given technology is very helpfull of radio frequency design. In bandwidth improvement of an equilateral trangularMsP antenna under differential exitation, the radition directivity of TM11 mode is atteched such a patch. For improved a lot of field distribution, Wang et al. (2017) [14] have presented an additional mod for both TM10and TM11 exited for radiation. Both virtual and measured result has been exhibit wide bandwidth and good presentation of radiation. Broker et al. (2016) [15] given a linearly polarized dual-band patch antenna resulting low cross-polarization and autonomous band control.Finelly, the resultsgives lower operating band and upper operating band is shifted up to $10 \%$ lastly.To minimize the cross-polarization, low loss is applied to integrate the antenna array and bring improvement in antenna array gain, Jing et al. (2015) [16] have presented a low temperature cofired ceramic (LTCC) process. This process has been used to fabricate and measure outcomes and found10$\mathrm{dB}$ of impedance and the gain of $18.62 \mathrm{~dB}$ at $61.5 \mathrm{GHz}$.

Trong et al. (2017) [17] proposed a center-shorted MsPmeschnism by which DC bias voltage; both resonance frequencies variedsimulataneously. Li et al. (2016) [18] given a vertically integrated differential filtering antenna that composed of a differential-fed MsP antenna with U-shaped differential resonator. The outcomes of [18] behave as frequency responce for both gain and return loss. In order to generate millimeterwave, Yao et al. (2016) [19] gave a Hermite-Gussian (HG) method and are formed by four inset-fed MsP element also with a microstripcorporate feeding network. Through [19] accuracy in measurement and simulation is achieved. Attaran et al. (2016) [20], described a Rotman lens method in which the length on the communication lines are not affected the progressive phase delay. Through [20], the complexity is minimized and performance parameters are maximized which finally gives low phase error of 0.450 in critical condition.

Zhang et al. (2016) [21] illustratedMsP antenna with the capabilities of bandwidth and harmonic suppression. Here, a pair of lemda/4 microstrio-line, wide band property can be obtained by making useful use of thr two resonances introduce by burning patch and non-radiating patch. The given prototype antenna is oprating at $4.9 \mathrm{GHZ}$ is designed and fabricated, higher-order radiating modes has been effectively cancelled. In Sun et al. (2016) [22], a proximity coupled cavity backed patch antenna is expressed for long range RFID tag. The patch structure also offers a way to tune the resonant frequency of the antenna. The given antenna is achieved a gain of $5.7 \mathrm{dBi}$. In order to provide support towards two distinct operating frequencies author Smyth et al. (2016) [23] have given a novel dual-band MsPantenna based on EBG itegrated into its radiating edges. Through this dual antenna radiation tracked at $2.4 \mathrm{GHz}$ and $5.0 \mathrm{GHz}$ frequency. Thus, in this paper the priority is given for designing an accurate computerized tool for MsPantenna and following utilized aanalytical approach which composed optimized synthesis of patterns for MsP antenna design.

\section{SYSTEM MODEL FOR MICROSTRIP PATCH (MsP) ANTENNA}

In order to get the geometrical topology for MsP antenna the architecture is shown in Figure 1. The core component such as height of MsP antenna (d1), width of MsP antenna (d2), width of metal ground slot aperture (w1), length of metal ground slot aperture (u1), clot center to patch center (v1), length of open end stub, terminates the feed (v2), chamber length of the input microstrip (wc) and microstrip signal trace 
width (wo) are considered. The starting point (Sp) of the topology is initiated as [Spx, Spy] to arrive to the patch element with Xpatch [], Ypatch [] a schematic shown in Figure 1.

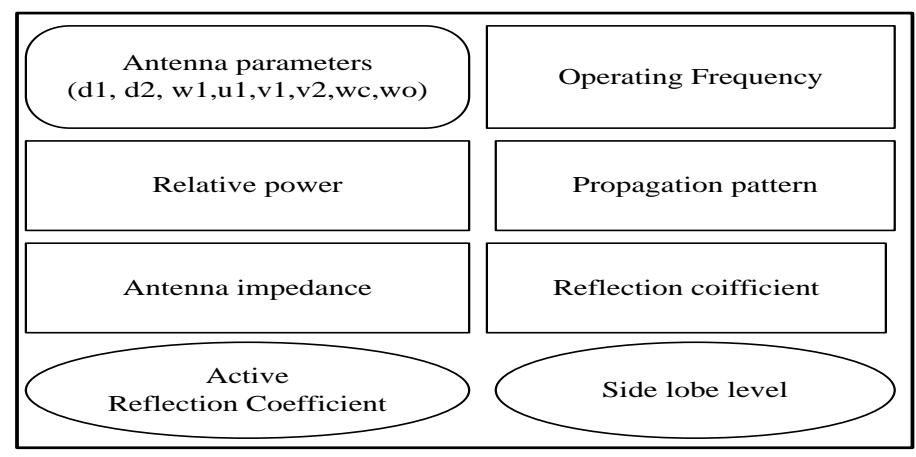

Figure 1. Architecture of proposed design

Here the information's of $\mathrm{d} 1, \mathrm{~d} 2, \mathrm{u} 1, \mathrm{v} 1, \mathrm{v} 2, \mathrm{wc}$ and wo are considered as input parameters. Later, the starting point $(\mathrm{Sp})$ is defined corresponding to the coordinates of Xpath and Ypath. The Sp can be obtained by,

$$
\begin{aligned}
& \text { Sp }=[\text { Spx }, \text { Spy }] \\
& {[\text { Xpatch }]=\left[\text { Spi }, \sum(\text { Spi }+\mathbf{d} 2), \sum(\text { Spi }+\mathbf{d} 2), \text { Spi, Spi }\right] \quad \text { at, } i=1} \\
& {[\text { Ypatch }]=\left[\text { Spi,Spi } \sum(\text { Spi }+\mathbf{d 1}), \sum(\text { Spi }+\mathbf{d 1}),(\text { Spi1) }] \text { at, } i=2\right.}
\end{aligned}
$$

Further, the center localization of the patch (Px, Py) is computed by using equation 4 .

$$
\begin{aligned}
& P x=\sum\left(\left(\operatorname{Spi}+\left(\frac{\mathbf{d} 2}{2}\right)\right), i=1\right. \\
& P y=\sum\left(\left(\operatorname{Spi}+\left(\frac{\mathbf{d} 1}{2}\right)\right), i=2\right.
\end{aligned}
$$

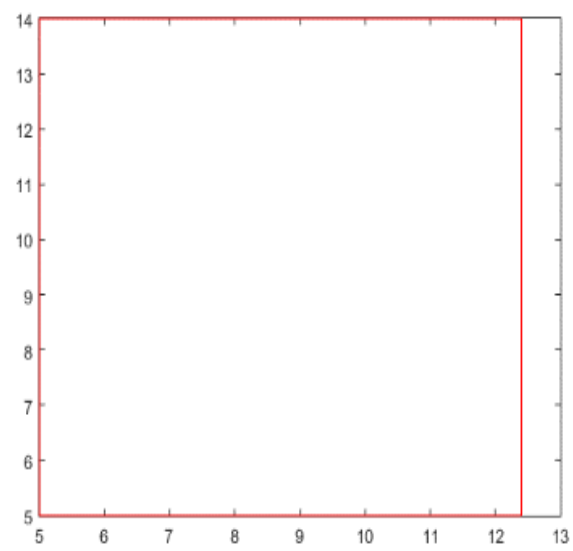

Figure 2. Patch with Xpatch []. Ypatch [] determined.

Based on these coordinates, a rectangle is plotted and for the same rectangle, patch center is

$[\mathrm{PxPy}] \leftarrow \mathrm{f}(\mathrm{Spi}, \mathrm{d} 1, \mathrm{~d} 2)$ at $\mathrm{i}=1,2$ 
Using this patch center, a center patch line is drawn.

$$
\begin{aligned}
& \text { i.e. }[p x-2 p x+v 1+v 2],[p y p y] \\
& {[p x p x],[2 p y+d 1 / 3+0.5]}
\end{aligned}
$$

Then the clot center $(\mathrm{Cc})$ is obtained, which is relative to the patch center.

$$
\mathrm{Cc}=([\mathrm{px}+\mathrm{v} 1 \mathrm{px}+\mathrm{v} 1],[\mathrm{py}-\mathrm{w} 0 / 2 \mathrm{py}+\mathrm{d} 1 / 3+0.5]
$$

The distance for right angled triangle $\left(x_{t}\right)$ is obtained by using equation 8 .

$$
\text { i.e. } x_{t}=\sqrt{w c^{2} / 2}
$$

Then slot aperture of metal ground is calculated for both the Xpath and Ypath using equation 9.

$$
\begin{aligned}
& \text { xmetal_ground }=[p x+v 1-w 1 / 2 p x+v 1+w 1 / 2 p x+v 1+w 1 / 2 p x+v 1-w 1 / 2 p x+v 1-w 1 / 2] \\
& \text { ymetal_ground }=[p y-u 1 / 2 p y-u 1 / 2 p y+u 1 / 2 p y+u 1 / 2 p y-u 1 / 2]
\end{aligned}
$$

Finally the labeling of the plot is done and outcome of the topology is shown in the Figure 3 .

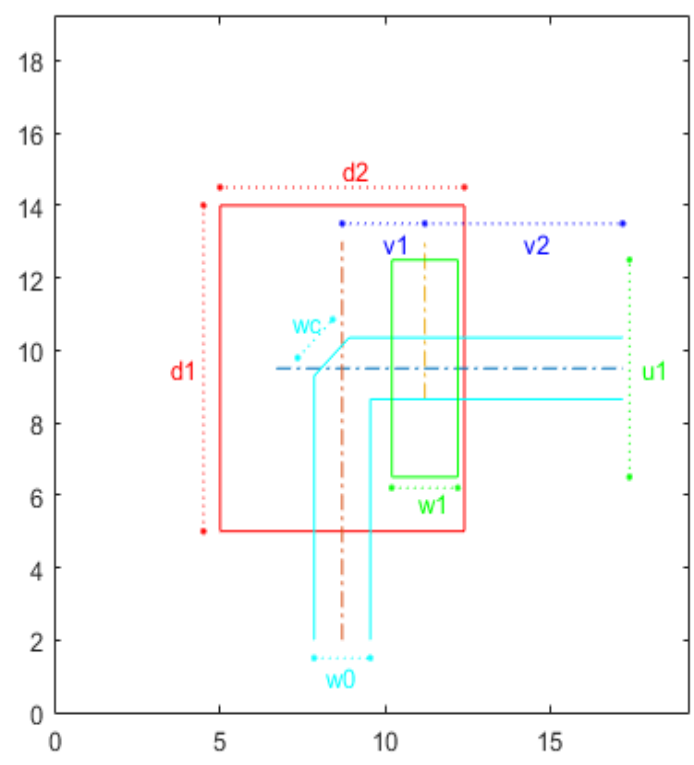

Figure 3. Default topology of MsP antenna

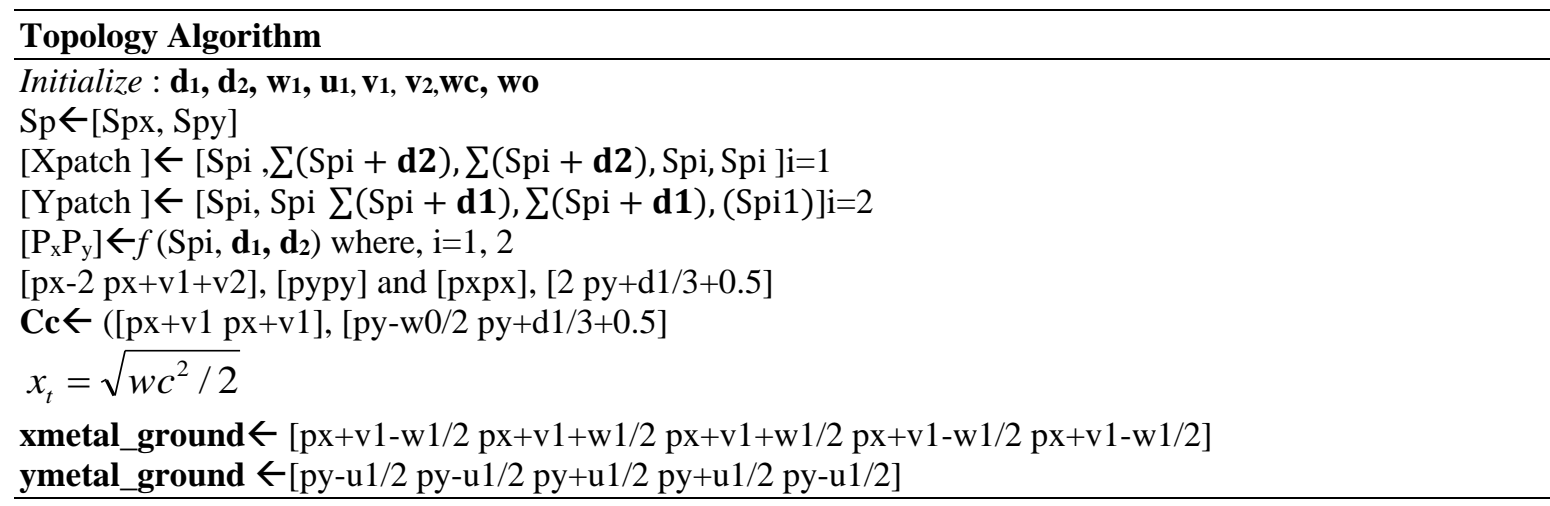




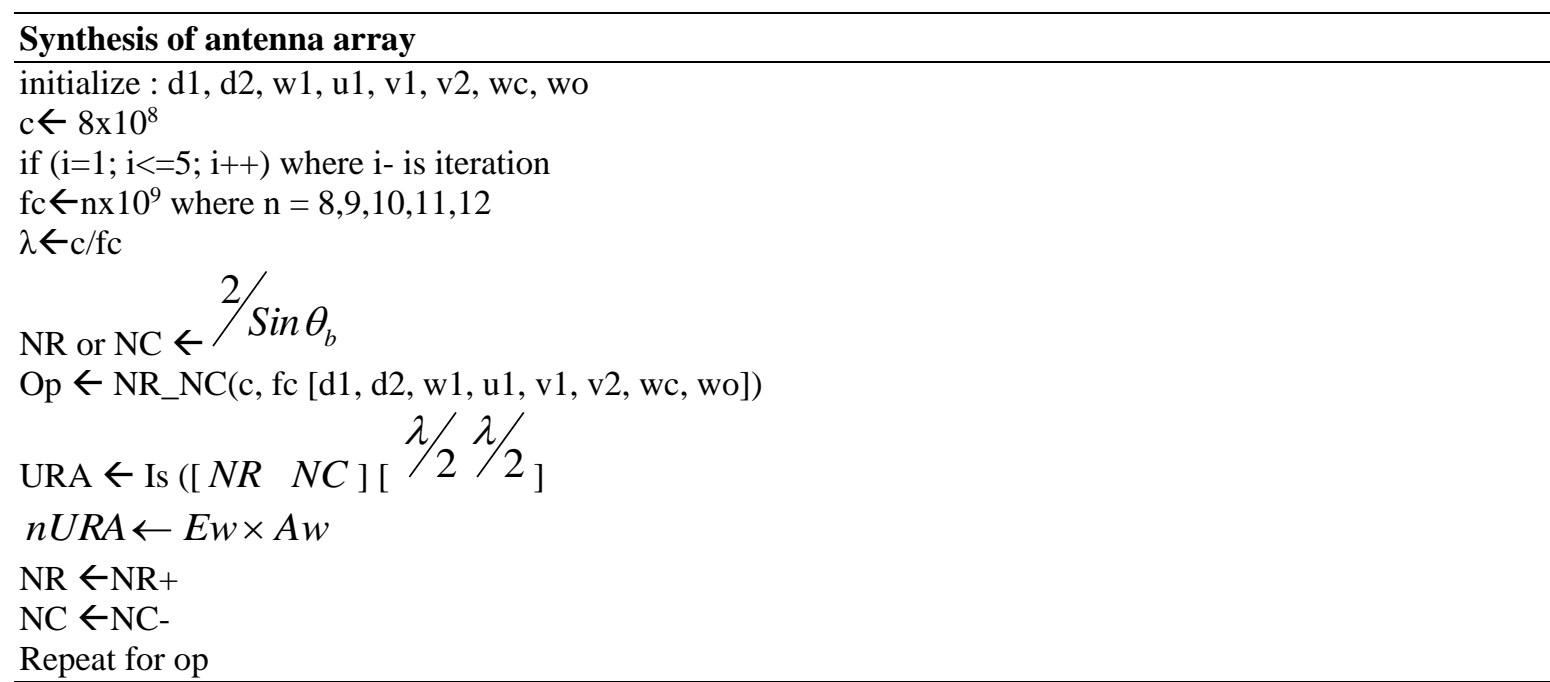

To compute the relative power, the parameters like d1, d2, w1, u1, v1, v2, wc, wo are initialized. Later, carrier frequency (fc) computed by using signal propagation speed (c). Further, wavelength $(\lambda)$ is calculated by dividing "c" with "fc".

$$
\text { i.e., } \lambda=\mathrm{c} / \mathrm{fc}
$$

The array size along with elevation and azimuth direction can be obtained by required beam width. For the half wavelength spacing, the number of elements along with certain direction can be given as;

$$
\mathrm{NR} \text { or } \mathrm{NC}=2 / \operatorname{Sin} \theta_{b}
$$

In equation 11 , the value of $\theta_{b}$ represents the beam width along that direction. The other parameters (Op) like azimuth cutoff and elevation cut off can obtain by following equation.

$$
\text { Op }=\text { NR_NC(c, fc }[\mathrm{d} 1, \mathrm{~d} 2, \mathrm{w} 1, \mathrm{u} 1, \mathrm{v} 1, \mathrm{v} 2, \mathrm{wc}, \mathrm{wo}])
$$

Then, the uniform rectangular array (URA) is considered as the integration of two separable uniform Line arrays (ULA) and designed the windows for both the elevation and azimuth direction through digital filer design methods. Then the URA developed by identical sensor elements can be given as:

$$
\text { i.e., } \mathrm{URA}=\mathrm{Is}\left(\left[\begin{array}{ll}
N R & N C
\end{array}\right][\lambda / 2 \lambda / 2]\right.
$$

In equation 13, Is indicates the identical sensor element. On assigning the weights to the array following equation 14 is obtained.

$$
\text { i.e., } n U R A=E w \times A w
$$

Where nURA indicates the new URA, Ew indicates the elevation weight and Aw represents the Azimuth weight. Later the comparison among the new URA and previous URA. In antenna technology the side lobes are the local maxima or lobes of the far field radiation pattern which are not the main lobes. Here, the side lobe level of the new URA is compared with the previous design. However, the new URA does not meet the requirements and hence trial and error method is applied to NR and NC parameters.

$$
\begin{aligned}
& \text { i.e., } \mathrm{NR}=\mathrm{NR}+ \\
& \mathrm{NC}=\mathrm{NC}-
\end{aligned}
$$

Then obtained values of NR and NC are updated to get the optimized design results. 


\section{RESULTS ANALYSIS}

For design of MsP antenna MATLAB is used and obtained results on execusion. The performance analysis of the design is compared with existing method. The following Figure 4, illustrates the beam pattern for looks directions ranging from $<-30^{\circ}$ to $0^{0}$ azimuth and elevation degrees and maintains null at $-40^{\circ}$.
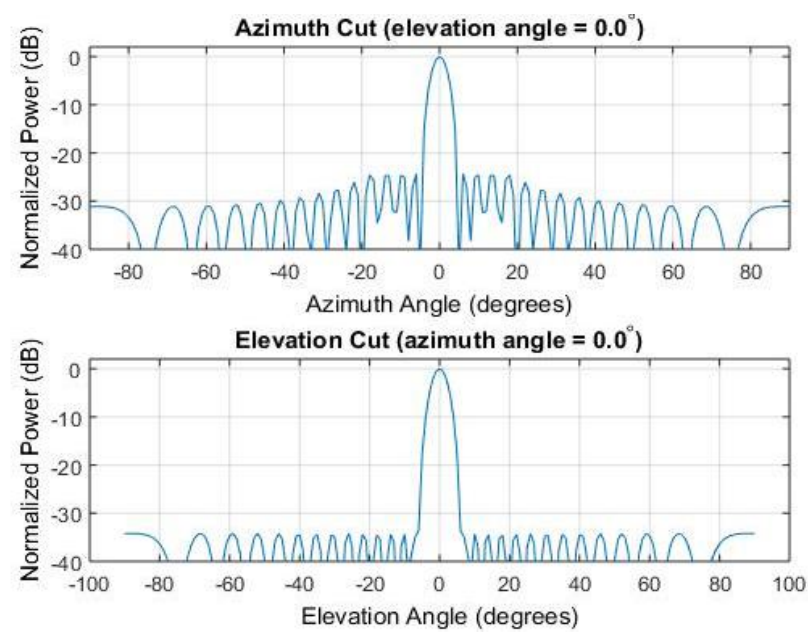

Figure 4. Beam pattern for azimuth and elevation degree

The array synthesis is represented in Figure 5 with respect to topology 1, 2, 4 and optimal topology by considering bandwidth. Here, the topology 1 array is just crossing the required bandwidth of patterns of topology 2, 4 and optimal topology. However, the side lobes of patterns bandwidth is higher that of desired pattern. This kind of side lobes can be optimized by utilizing windowing operations to array. If URA is the combination of two different uniform linear arrays (ULA), then thedesign of window can be performed separately in both elevation and azimuth directions by utilizing filter designing models. The Figure 4 gives the side lobe level compared with different topologies and is found that side lobe level of optimal topology is less than topology 1 , topology 2 and topology 4.

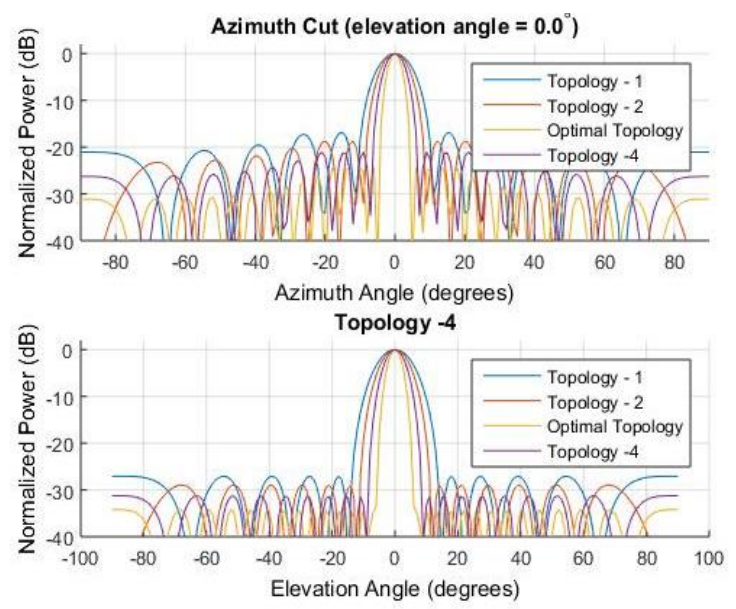

Figure 5. Beam patterns synthesis with different methods

The $3 \mathrm{D}$ radiation patterns are composed of symmetries for both azimuth and elevation cuts. Hence, the patterns are acquired through URA. The Figure 6 indicates that no energy is radiated in reverse to back of array in which the bandwidth and side lobe level of synthesized pattern are resulted with desired specification and is considered as $3 \mathrm{D}$ pattern synthesis. 


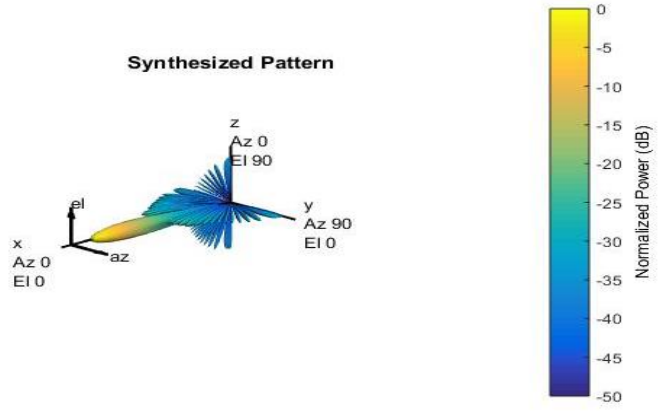

Figure 6. design pattern (3D) syntehesis

The Figure 7 represents the MsP antenna over the frequency band. In this, the resistance and reactance varyas frequency varies. This variation can be seen that the reactance value is negative before the resonance and the same value is positive after the resonance and this reactance is considered as"series resonance". If impedance curve varies from positive to negative reactance and is considered as "parallel resonance". Both the resistance and reactance are fully different as resistance which is not depend on frequency while reactance does. The resistance does not cause phase shift while reactance causes phase shift of $90^{\circ}$ among voltage and current. In Figure 7, resistance remains at positive value and reactance stays at negative value during resonance and reaches positive after resonance.

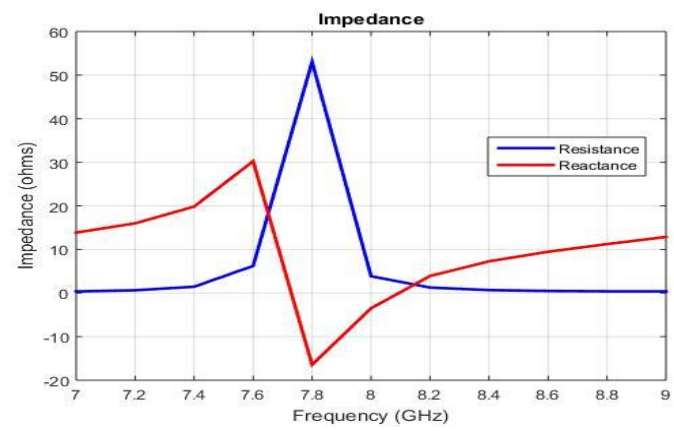

Figure 7. Antenna performance over frequency band

The antenna reflection coefficient is shown in Figure 8 which is the relative fraction of the incident Radio frequency (RF) power and is reflected back because of impedance mismatch. The impedance mismatch is the difference among the antenna input impedance and the transmission line characteristic or reference impedance. The reflection coefficient is represented as operating bandwidth of antenna. The antenna bandwidth is the frequency band on which the magnitude of reflection coefficient $<-10 \mathrm{~dB}$.

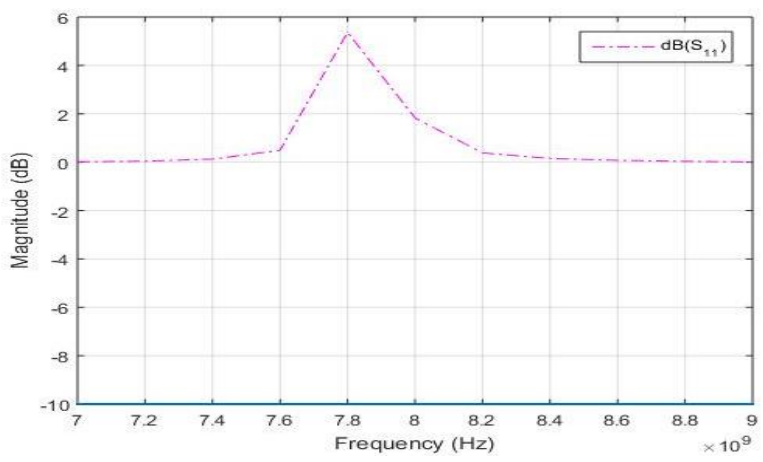

Figure 8. Analysis offrequency with respect to magnitude 
The active reflection coefficients analysis with four different topology are compared corresponding to frequency are shown Figure 9, where optimal topology acquired positive value than other topology1, topology2 and topology4.

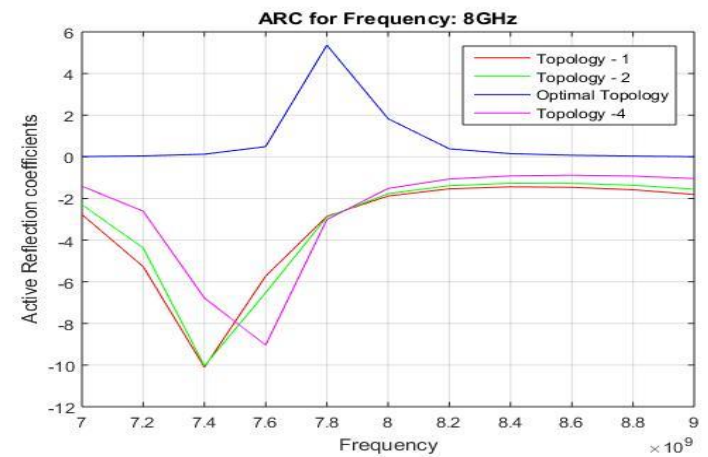

Figure 9. Active reflection coefficient Vs frequency

The Figure 10 represents the array side lobe level for different methods. The side lobes are the far field radiation pattern and are not considered as main lobes. The side lobe level increases with decreases in the bandwidth. In order to get a significant array pattern, the side lobe level value must be higher. From the Figure 10, it is found that the optimal topology, topology1, topology 2and topology 4exhibits 24.3109, $16.8963,18.7218$, and 21.0294 respectively. Thus, the optimal topology acquires lower antenna cost with higher value of side lobe level.

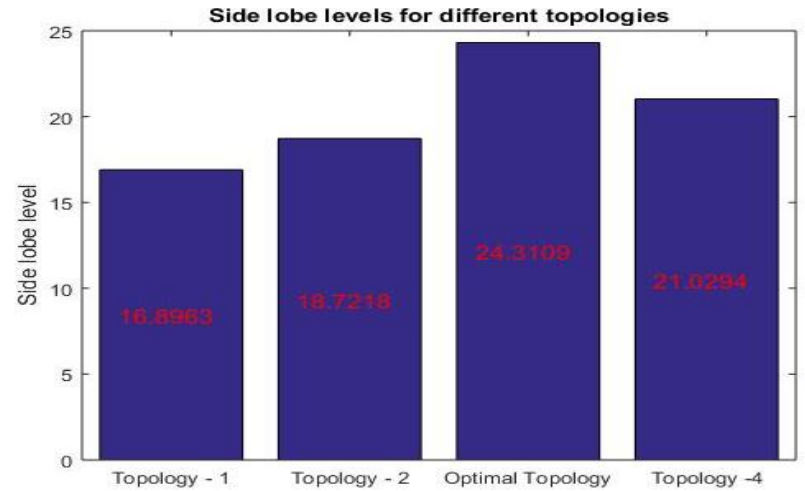

Figure 10. Side lobe levels for different methods

\section{CONCLUSION}

This paper introduces ananalytical approach to perform the simulation of linear MsP antenna design. The results of the design were considered with respect to beam patterns synthesis, design pattern (3D) syntehesis, antennaperformance over frequency band, and analysis offrequency with respect to magnitude, active reflection coefficient Vs frequency and Side lobe levels for different methods. The outcomes of the approach allow significantly controlling both the radiation as well as reflection coefficient through the element geometry design and identical sensor element. The analytical approach come up with minimized the cost of antenna to low side lobe level corresponding to some EMI of array antenna.

\section{REFERENCES}

[1] C. A. Balanis, "Antenna theory," 3rd ed., Wiley Inter science, 2005.

[2] R. J. Mailloux, "Phased array antenna handbook," 2nd ed., Artech House, 2005.

[3] S. Koziel and S. Ogurtsov, "Antenna design using variable fidelity electromagnetic simulations," Int. J. Applied Electromagnetics and Mechanics, vol. 43, pp. 169-183, 2013. 
[4] S. Koziel, et al., "Variable-fidelity electromagnetic simulations and co-kriging for accurate modeling of antennas," IEEE Transactions on Antennas and Propagation, vol/issue: 61(3), pp. 1301-1308, 2013.

[5] M. M. Khodier and C. G. Christodoulou, "Linear array geometry synthesis with minimum sidelobe level and null control using particle swarm optimization," IEEE Trans. Antennas Prop., vol/issue: 53(8), pp. 2674-2679, 2005.

[6] F. J. A. Pena, et al., "Genetic algorithms in the design and optimization of antenna array patterns," IEEE Trans. Antennas Prop., vol. 47, pp. 506-510, 1999.

[7] D. W. Boeringer, et al., "A simultaneous parameter adaptation scheme for genetic algorithms with application to phased array synthesis," IEEE Trans. Antennas Prop., vol. 53, pp. 356-371, 2005.

[8] R. J. Kavitha and H. S. Aravinda, "Reviewing the Effectiveness of Contribution of Microstrip Antenna in the Communication System," Open Journal of Antennas and Propagation, vol/issue: 5(02), pp. 47, 2017.

[9] X. Tang, et al., "Design of a Wideband Circularly Polarized Strip-Helical Antenna with a Parasitic Patch," IEEE Access, vol. 4, pp. 7728-7735, 2016.

[10] A. A. Salih and M. S. Sharawi, "A Dual-Band Highly Miniaturized Patch Antenna," IEEE Antennas and Wireless Propagation Letters, vol. 15, pp. 1783-1786, 2016.

[11] J. D. Zhang, et al., "CP patch antenna with controllable polarisation over dual-frequency bands," IET Microwaves, Antennas \& Propagation, 2016.

[12] A. Katyal and A. Basu, "Analysis and optimisation of broadband stacked MsAs using transmission line model," IET Microwaves, Antennas \& Propagation, 2016.

[13] C. Hannachi and S. O. Tatu, "Performance comparison of $60 \mathrm{GHz}$ printed patch antennas with different geometrical shapes using miniature hybrid microwave integrated circuits technology," IET Microwaves, Antennas \& Propagation, 2016.

[14] J. Wang, et al., "Bandwidth Enhancement of a Differential-Fed Equilateral Triangular Patch Antenna via Loading of Shorting Posts," IEEE Transactions on Antennas and Propagation, vol/issue: 65(1), pp. 36-43, 2017.

[15] D. E. Brocker, et al., "Miniaturized Dual-band Folded Patch Antenna with Independent Band Control Utilizing an Interdigitated Slot Loading," IEEE Transactions on Antennas and Propagation, 2016.

[16] H. Jin, et al., "High-gain low-cross-polarization 60-GHz LTCC patch antenna array with differential-fed and softsurface structures," Microwave Conference (APMC), 2015 Asia-Pacific, vol. 1, 2015.

[17] N. N. Trong, et al., "A frequency-and pattern-reconfigurable center-shorted MsA," IEEE Antennas and Wireless Propagation Letters, vol. 15, pp. 1955-1958, 2016.

[18] L. Li and G. Liu, "A Differential MsAWith Filtering Response," IEEE Antennas and Wireless Propagation Letters, vol. 15, pp. 1983-1986, 2016.

[19] H. Yao, et al., "Patch Antenna Array for the Generation of Millimeter-Wave Hermite-Gaussian Beams," IEEE Antennas and Wireless Propagation Letters, vol. 15, pp. 1947-1950, 2016.

[20] A. Attaran, et al., "60 GHz Low phase error rotman lens combined with wideband MsA array using LTCC technology," IEEE Transactions on Antennas and Propagation, vol/issue: 64(12), pp. 5172-5180, 2016.

[21] J. D. Zhang, et al., "A Compact Microstrip-Fed Patch Antenna with Enhanced Bandwidth and Harmonic Suppression," IEEE Transactions on Antennas and Propagation, vol/issue: 64(12), pp. 5030-5037, 2016.

[22] H. Sun, et al., "Proximity Coupled Cavity Backed Patch Antenna for Long Range UHF RFID Tag," IEEE Transactions on Antennas and Propagation, vol/issue: 64(12), pp. 5446-5449, 2016.

[23] B. P. Smyth, et al., "Dual-Band Microstrip Patch Antenna Using Integrated Uniplanar Metamaterial-Based EBGs," IEEE Transactions on Antennas and Propagation, vol/issue: 64(12), pp. 5046-5053, 2016.

\section{BIOGRAPHIES OF AUTHORS}

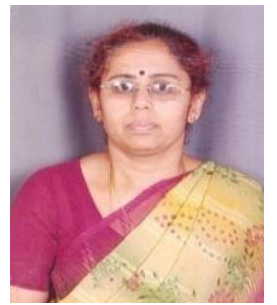

Kavitha R J working as a research scholar at Visvesvaraya Technological University, Belagavi has around 5 research papers to her credit. She has served in different organizations and has around 16+ years of experience. She has completed her BE from Mysore University and MTechfrom Visvesvaraya Technological University in 2008. She has been instrumental in the process of Accreditation work.

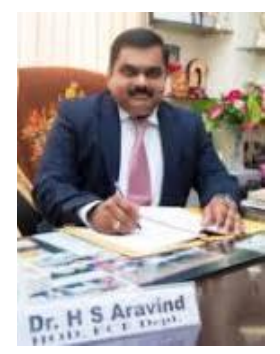

Dr. Aravind HS, MTech, Ph.D., is a professor and head of Electronics and Communication engineering department at JSSATE, Bengaluru.He has more than 50international/ national papers to his credit. He has served in various organisations in different levels. He has completed his Doctorate from Visvesvaraya Technological University and is specialized in the area of fault tolerance, signal processing. 